\title{
New Applications in Power Electronics Based on SiC Power Devices
}

Hervé Morel', Dominique Bergogne', Dominique Planson', Bruno Allard' and Régis Meuret ${ }^{2}$

${ }^{1}$ AMPERE lab. INSA Lyon, Bat Leonard de Vinci, 69621 Villeurbanne, France

${ }^{2}$ Hispano-Suiza, SAFRAN group, BP 42, 77551 Moissy Cramaye, France

Herve.Morel@insa-lyon.fr, Regis.Meuret@hispano-suiza-sa.com

Keywords: SiC devices, JFET, Power Electronics, High Temperature, High Voltage.

\section{Introduction}

Nowadays some silicon carbide, $\mathrm{SiC}$, power devices are commercialized like Schottky diodes. Moreover power JFETs are available as industrial samples. The two latter devices enable to build most of power electronic converters. One main issue is for which applications silicon carbide devices can advantageously replace classical silicon power semiconductor devices ?

The first class of applications for $\mathrm{SiC}$ power devices are applications for which the use of silicon power devices are not possible. The second class of applications for $\mathrm{SiC}$ devices correspond to systems where mass reduction is a big issue, i.e. for embedded systems like aeronautic systems.

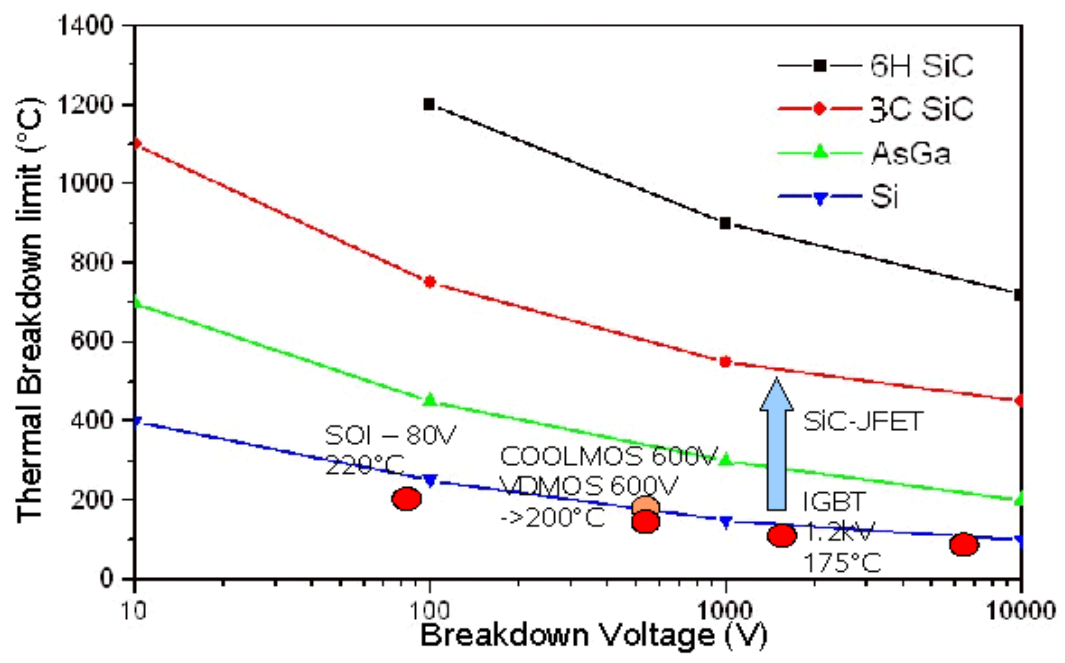

Fig. 1: Temperature limit versus voltage limit of semiconductor devices

Fig. 1 illustrates the first case of applications. The figure yields the thermal breakdown limit versus the breakdown voltage of an infinite plane junction in various semiconductor. These are theoretical limits. Spots correspond to industrial silicon power devices. It is clear that there are very near from the theoretical limit. The particular case of the CoolMOS is because it is not a junction but a superjunction. Concerning high voltage devices the thermal limit is too a major constraint for silicon devices.

So, clearly, applications becoming possible because of new SiC power devices are high temperature and/or high voltage applications.

However, most of recent studies have shown that the use of SiC devices does not consist in a simple replacement of silicon devices by silicon carbide devices. Indeed a new design of the new power electronic systems taking advantage of the properties of the new SiC power devices are required.

\section{High Temperature Applications}

Several recent studies address high temperature applications. For instance the More Electric 
Aircraft, MEA, demands high temperature power electronics for electrification of actuators mounted on the aircraft engine. In such applications, the ambient temperature, i.e. the engine temperature, may reach $200^{\circ} \mathrm{C}$. Due to self heating of the power semiconductor devices, the "junction" temperature may reach $250^{\circ} \mathrm{C}$ even $300^{\circ} \mathrm{C}$. Silicon power device are not applicable because the voltage rate is about $1200 \mathrm{~V}$ and thermal breakdown is reached as shown in Fig. 1.

Several studies have shown the operation of an inverter cooled at $300^{\circ} \mathrm{C}$. The industrial goal is $200^{\circ} \mathrm{C}$ of ambient temperature.

Remaining questions are,

- the reliability of the system, i.e. mainly at the packaging level,

- the converter structure (diode-less or not) to optimize efficiency and reliability,

- the driver structure, including protection operation (taking advantage of the high limit of $\mathrm{SiC}$ thermal breakdown, short-circuit and avalanche robustness of power device are very good).

- high temperature passive devices

Several studies in the world are under development with different similar objectives. Most of them are based on SiC JFET because there are almost available at the industrial level. However research devices like MOSFET, and high temperature Schottky diodes, or other wide-band semiconductor like GaN may contribute to medium or long term alternatives to the current studies.

\section{High Voltage Applications}

Such applications are medium terms applications because high voltage devices are not ready for industrial applications. However $10 \mathrm{kV}$ demonstrators have been developed, $15 \mathrm{kV}$ demonstrators are under development even $30 \mathrm{kV}$ and further may be soon obtained.

The interest of such devices are enormous because they enable to

- replace heavy $50 \mathrm{~Hz}$ high voltage electrical transformers by high voltage power electronics.

- replace AC-current distribution networks by DC-current distribution networks and eliminating numerous associated problems like instability (wind-power farm ...) even blackout. That enables an easier integration of renewable energy sources (Photovoltaic, Wing power ...) and remove the need of $50 \mathrm{~Hz}$ electric transformers.

- improve the high voltage system protection with very fast active protection systems

The main issues of these new applications are,

- availability of high bipolar diodes and switches (BJT, Thyristors, ...)

- electrical insulation of the high voltage converters (packaging, passivation, ...)

- thermal cooling of the high voltage converters

- highly insulated driver for high voltage converters even multi-level converters.

\section{Power Electronics in the Embedded Systems}

The mass reduction and medium high temperature are applications for $\mathrm{SiC}$ devices, but in such a case the interest of $\mathrm{SiC}$ devices have to be proved even from the economic point of view, because silicon alternatives exist. However, the use of high junction temperature to reduce the mass of the thermal cooling system is a way to be explored with the reliability of such new systems.

\section{References}

[1] D. Bergogne, P. Bevilacqua, S. M'Rad, D. Planson, H. Morel, B. Allard, O. Brevet, « $300^{\circ} \mathrm{C}$ operating junction 
temperature inverter leg investigations » EPE'05, Dresden, pp. 1-8.

[2] Jun Wang, Liyu Yang, Tiefu Zhao, Alex Q. Huang, Characteristics of $10 \mathrm{kV} \mathrm{SiC} \mathrm{MOSFET} \mathrm{and} \mathrm{PIN} \mathrm{Diode} \mathrm{and}$ Their Application Prospect in High Voltage High Frequency DC/DC Converter, IEEE PESC'0, pp. 72-77 